\title{
HYPERSURFACES WITH CONSTANT MEAN CURVATURE IN THE COMPLEX HYPERBOLIC SPACE
}

\author{
SUZANA FORNARI, KATIA FRENSEL, AND JAIME RIPOLL
}

\begin{abstract}
A classical theorem of A. D. Alexandrov characterized round spheres is extended to the complex hyperbolic space $\mathbf{C H}^{2}$ of constant holomorphic sectional curvature. A detailed description of the horospheres and equidistant hypersurfaces in $\mathbf{C H}^{2}$ determining in particular their stability, is also given.
\end{abstract}

\section{INTRODUCTION}

A classical theorem due to A. D. Alexandrov [A] proves that the geodesic spheres are the only compact embedded hypersurfaces with constant mean curvature in a simply connected space of constant curvature (with the additional hypothesis of the hypersurface being contained in a hemisphere in the spherical case). Since in a two point homogeneous space the geodesic spheres are homogeneous hypersurfaces and therefore with constant mean curvature, it is natural to ask if Alexandrov's Theorem can be extended to these spaces. We answer here this question affirmatively for the complex hyperbolic space. For simplicity, we work in the 2-dimensional (complex) case. We prove

Theorem 3.3. Let $M$ be a compact, embedded hypersurface with constant mean curvature of the complex hyperbolic space $\mathbf{C H}^{2}$. Then $M$ is a geodesic sphere.

The simplest examples of complete hypersurfaces with constant mean curvature in $\mathbf{C H}^{2}$ are the geodesic spheres, horospheres and equidistant hypersurfaces. J. L. Barbosa, M. P. do Carmo and J. Eschenburg proved that the geodesic spheres are stable (Theorem 1.4 of [BdoCE]). We determine here the stability of the horospheres and equidistant hypersurfaces of $\mathbf{C H}^{2}$. We recall that a horosphere is defined as the limit of the geodesic spheres which pass through a given point of the space and whose centers tend to infinity along a geodesic of the space.

As it happens in the real hyperbolic space, we prove here that the horospheres in $\mathbf{C H}^{2}$ are all stable. We also give a detailed description of them. We prove

Theorem 4.3. The horospheres of the complex hyperbolic space $\mathbf{C H}^{2}$ are hypersurfaces with constant mean curvature equal to 4/3 and are all stable. Furthermore, they are the orbits of the Heisenberg group (a 3-dimensional noncommutative nilpotent Lie group) which acts by isometries on $\mathbf{C H}^{2}$ without fixed points

Received by the editors August 20, 1990 and, in revised form, July 3, 1991.

1991 Mathematics Subject Classification. Primary 53C42; Secondary 53C40. 
(therefore, the horospheres inherit a natural Lie group structure). In particular, the horospheres are (extrinsically) homogeneous submanifolds of $\mathbf{C H}^{2}$. Any two horospheres of $\mathrm{CH}^{2}$ are congruent.

The equidistant hypersurfaces are defined in the following way: the hyperbolic plane $\mathbf{H}^{2}$ can be isometrically embedded in a unique way (up to congruences) as a totally geodesic submanifold of $\mathbf{C H}^{2}$ (this follows from the characterization of the totally geodesics submanifolds of a symmetric space). Given $c>0$, an equidistant hypersurface $P_{c}$ is defined as the boundary of the tubular neighborhood with radius $\sinh ^{-1}(c)$ of $\mathbf{H}^{2}$. In $\S 5$ we give a detailed description of such hypersurfaces. In particular, we prove

Theorem 5.3. An equidistant hypersurface $P_{c}$ in $\mathbf{C H}^{2}$ is a homogeneous hypersurface with constant mean curvature $\left(1+4 c^{2}\right) /\left(3 c \sqrt{1+c^{2}}\right)$. It is stable if $c \geq \sqrt{2} / 2$ and unstable otherwise. Two equidistant hypersurfaces are congruent iff they have the same mean curvature.

We remark that the function $\left(1+4 c^{2}\right) /(c \sqrt{1+c})$ attains its minimum absolute value at $c=\sqrt{2} / 2$, that is, $P_{\sqrt{2} / 2}$ is the equidistant hypersurface whose mean curvature is the smallest one.

In the next section, we obtain some basic facts about the Riemannian Geometry of $\mathbf{C H}^{2}$ necessary for proving the above theorems.

\section{Preliminaries}

2.1 The "Hopf fibration" of $\mathbf{C H}^{2}$. On $\mathbf{C}^{3} \equiv \mathbf{R}^{6}$ consider the indefinite scalar product

$$
\langle z, w\rangle:=\operatorname{Re}\left(-z_{0} \bar{w}_{0}+z_{1} \bar{w}_{1}+z_{2} \bar{w}_{2}\right)
$$

where $z=\left(z_{0}, z_{1}, z_{2}\right)$ and $w=\left(w_{0}, w_{1}, w_{2}\right)$ are points in the complex vector space $\mathbf{C}^{3}$. The set

$$
\mathbf{Q}^{5}:=\left\{z \in \mathbf{R}^{6} \mid\langle z, z\rangle=-1\right\}
$$

is a 5-dimensional submanifold of $\mathbf{C}^{3}$ and inherits a Lorentzian metric with constant sectional curvature -1 . The group $S^{1}=\left(e^{i \theta}\right)$ of complex numbers with modulus 1 acts freely on $\mathbf{Q}^{5}$ by complex multiplication so that $\mathbf{Q}^{5} / S^{1}$ is a differentiable manifold. The orbits $\alpha(\theta)=e^{i \theta} z$ for $z \in \mathbf{Q}^{5}$ of $S^{1}$ are timelike since

$$
\left\langle\alpha^{\prime}(\theta), \alpha^{\prime}(\theta)\right\rangle=\left\langle i e^{i \theta} z, i e^{i \theta} z\right\rangle=-1 .
$$

The Complex Hyperbolic Space $\mathbf{C H}^{2}$ can be defined as the quotient $\mathbf{Q}^{5} / S^{1}$ endowed with the Riemannian metric that makes the projection

$$
\pi: \mathbf{Q}^{5} \rightarrow \frac{Q^{5}}{S^{1}}
$$

a semi-Riemannian submersion.

2.2 The sectional curvatures of $\mathbf{C H}^{2}$. Let $X, Y$ be two orthonormal vector fields on $\mathbf{C H}^{2}$ and $\widetilde{X}, \widetilde{Y}$ be the horizontal lifts of $X$ and $Y$ on $\mathbf{Q}^{5}$, respectively. Denote by $K(X, Y)$ and $\widetilde{K}(\widetilde{X}, \widetilde{Y})$ the sectional curvatures of $\mathbf{C H}^{2}$ and $\mathbf{Q}^{5}$, respectively. The following formula is a straightforward extension to semi-Riemannian submersions of a result found in O'Neill [O1]. We have

$$
K(X, Y)=\tilde{K}(\tilde{X}, \tilde{Y})+3\left\langle\tilde{A}_{\widetilde{X}} \tilde{Y}, A_{\widetilde{X}} \tilde{Y}\right\rangle
$$


where $\tilde{A}$ is a tensor that reverses the horizontal and vertical subspaces of the projection $\pi$. Since the last subspace has real dimension 1 and, for a fixed $\widetilde{X}$, $\widetilde{A}_{X}$ is surjective, it follows that for any number $a, 0 \leq a^{2} \leq 1$, there exists $\tilde{Y}$ with $\langle\widetilde{Y}, \widetilde{Y}\rangle=1$ such that

$$
\left\langle\widetilde{A}_{\widetilde{X}} \tilde{Y}, \tilde{A}_{\widetilde{X}} \tilde{Y}\right\rangle=-a^{2} \geq-1 .
$$

Then $K(X, Y)=-1+3\left\langle\tilde{A}_{\widetilde{X}} \tilde{Y}, \tilde{A}_{\widetilde{X}} \tilde{Y}\right\rangle$ satisfies $-4 \leq K(X, Y) \leq-1$.

2.3 The $S^{1}$-orbits in $\mathbf{Q}^{5}$. Let $\alpha(\theta)=e^{i \theta} z$ be the $S^{1}$-orbit of a point $z \in \mathbf{Q}^{5}$. Then

$$
\alpha^{\prime \prime}(\theta)=-e^{i \theta} z=-\alpha(\theta)
$$

that is, $\widetilde{\nabla}_{\alpha^{\prime}} \alpha^{\prime}=0$, where $\tilde{\nabla}$ denotes the covariant derivative in $\mathbf{Q}^{5}$. It follows that the $S^{1}$-orbits are totally geodesic 1-dimensional submanifolds of $\mathbf{Q}^{5}$.

2.4 The mean curvature of a hypersurface of $\mathrm{CH}^{2}$ and of its lift in $\mathbf{Q}^{5}$. Let $M$ be a hypersurface in $\mathbf{C H}^{2}$ and denote by $\widetilde{M}$ its lift in $\mathbf{Q}^{5}$, that is, $\pi(\widetilde{M})=M$. Clearly, $\widetilde{M}$ is a $S^{1}$-invariant hypersurface of $\mathbf{Q}^{5}$. Let $p$ be a point in $\widetilde{M}$ and let $\widetilde{N}$ be an unitary $S^{1}$-invariant vector field normal to $\widetilde{M}$ around $p$. Set $N:=\pi_{*}(\tilde{N})$. Then $N$ is a unitary vector field normal to $M$ around $\pi(p)$. Let $\widetilde{H}$ and $H$ be the mean curvature of $\widetilde{M}$ at $p$ and of $M$ at $\pi(p)$ determined by $\tilde{N}$ and $N$, respectively. Then we have

Lemma 2.4. $H=\frac{4}{3} \tilde{H}$.

Proof. Consider an orthonormal frame $\left\{e_{i}\right\}, i=1,2,3$, tangent to $M$ in a neighborhood of $\pi(p)$. By definition

$$
H=\frac{1}{3} \sum_{i=1}^{3}\left\langle\nabla_{e_{i}} e_{i}, N\right\rangle
$$

where $\nabla$ is the Riemannian connection in $\mathbf{C H}^{2}$. Let $\tilde{e}_{i}$ be the horizontal lift of $e_{i}$ and $\bar{e}_{0}$ the unitary tangent vector to the fiber of $\pi$. It is obvious that $\bar{e}_{0}, \ldots, \bar{e}_{3}$ is an orthonormal frame tangent to $\widetilde{M}$ in a neighborhood of $p$. If $\widetilde{\nabla}$ denotes the semi-Riemannian connection of $Q^{5}$, then $\widetilde{\nabla}_{e_{0}} e_{0}=0$ by 2.3 , and $\left\langle\nabla_{e_{i}} e_{i}, N\right\rangle=\left\langle\widetilde{\nabla}_{\tilde{e}_{i}} \tilde{e}_{i}, \tilde{N}\right\rangle$, by O'Neill's formulae for a submersion [O1].

Then

$$
H=\frac{1}{3} \sum_{i=1}^{3}\left\langle\widetilde{\nabla}_{\tilde{e}_{i}} \tilde{e}_{i}, \tilde{N}\right\rangle=\frac{4}{3} \tilde{H} .
$$

We recall that

$$
\widetilde{H}=\frac{1}{4} \sum_{i=1}^{4} \varepsilon_{i}\left\langle\widetilde{\nabla}_{\tilde{e}_{i}} \tilde{e}_{i}, \tilde{N}\right\rangle,
$$

where $\varepsilon_{i}=\left\langle\tilde{e}_{i}, \tilde{e}_{i}\right\rangle= \pm 1$. In our case, $\left\langle\tilde{e}_{0}, \tilde{e}_{0}\right\rangle=-1$ and $\left\langle\tilde{e}_{i}, \tilde{e}_{i}\right\rangle=1, i=$ $1,2,3$.

2.5 Stability of hypersurfaces with constant mean curvature. Let $x: M^{n} \rightarrow$ $\bar{M}^{n+1}$ be an immersion between Riemannian manifolds, $x$ with constant mean curvature. Let $D \subset M$ be a relatively compact domain with smooth boundary 
$\partial D$. Set $\bar{D}:=D \cup \partial D$. Denote by $\mathscr{F}_{D}$ the set of all piecewise smooth functions $f: \bar{D} \rightarrow \mathbf{R}$ that satisfy the conditions

$$
\left.f\right|_{\partial D} \equiv 0, \quad \int_{D} f d M=0 .
$$

The domain $D$ is stable iff $\mathscr{T}_{D}^{\prime \prime}(0)(f) \geq 0$, for any $f \in \mathscr{F}_{D}$, where

$$
\mathscr{T}_{D}^{\prime \prime}(0)(f)=\int_{D}\left\{|\operatorname{grad}(f)|^{2}-\left(\operatorname{Ricc}(N)+\|B\|^{2}\right) f^{2}\right\} d M .
$$

Here grad is the gradient of $D$ in the induced metric, $\|B\|$ is the norm of the second fundamental form of $x$ and $\operatorname{Ricc}(N)$ is the Ricci curvature of $\bar{M}$ in the direction of the normal $N$ of $x$. Recall that, at a point $p$ in $M$, $\operatorname{Ricc}(N)=\sum_{i=1}^{n-1} K\left(N, e_{i}\right)$, where $e_{1}, \ldots, e_{n-1}, N$ is an orthonormal basis of $T_{p}(M)$ with $N$ normal to $M$ and $K$ denotes the sectional curvature of $\bar{M}$ at $p$.

The immersion $x$ is stable if any relatively compact domain $D \subset M$ is stable.

In our case, we will study stability of hypersurfaces of $\mathbf{C H}^{2}$. We remark that there exists an obvious semi-Riemannian version of Proposition 4.3 of [BdoCE], which reads: $M$ is stable in $\mathbf{C H}^{2}$ iff $\widetilde{M}=\pi^{-1}(M)$ is " $S^{1}$-stable" in $\mathbf{Q}^{5}$, that is, iff

$$
\int_{D}\left\{|\operatorname{grad}(f)|^{2}-\left(\operatorname{Ricc}(\widetilde{N})+\|\widetilde{B}\|^{2}\right) f^{2}\right\} d \widetilde{M} \geq 0
$$

for all relatively compact and $S^{1}$-invariant domains $D \subset \widetilde{M}$ and for all $f$ in $\mathscr{F}_{D}$ with the following property: $f\left(e^{i \theta} p\right)=f(p), e^{i \theta} \in S^{1}$ and $p \in \widetilde{M}$.

The vector field $\widetilde{N}$ considered above is a unitary normal vector field of $\widetilde{M}$ in $\mathbf{Q}^{5}$ and $\widetilde{B}: T_{p}(\widetilde{M}) \rightarrow T_{p}(\widetilde{M})$ is the linear symmetric transformation associated to the second fundamental form $\alpha$ of $\widetilde{M}$ in $\mathbf{Q}^{5}$, that is, if $\left\{e_{i}\right\}_{i=1}^{4}$ is an orthonormal basis of $T_{p}(\widetilde{M}), \varepsilon_{i}=\left\langle e_{i}, e_{i}\right\rangle= \pm 1$, then $\left\langle\widetilde{B}\left(e_{i}\right), e_{j}\right\rangle=$ $\left\langle\alpha\left(e_{i}, e_{j}\right), \widetilde{N}\right\rangle$. The norm $\|\widetilde{B}\|$ at $p \in \widetilde{M}$ is defined by

$$
\|\widetilde{B}\|_{p}^{2}=\sum_{i=1}^{4} \varepsilon_{i}\left(\widetilde{B}\left(e_{i}\right), \widetilde{B}\left(e_{i}\right)\right\rangle_{p}=\sum_{i, j=1}^{4} \varepsilon_{i} \varepsilon_{j}\left\langle\alpha\left(e_{i}, e_{j}\right), \widetilde{N}\right\rangle^{2} .
$$

We observe that $\|\widetilde{B}\|$ is independent of the orthonormal basis $\left\{e_{i}\right\}$. This fact is a consequence of the following general result:

Lemma 2.5. Let $V$ be an n-dimensional vector space with a nondegenerate symmetric bilinear form $\langle\cdot, \cdot\rangle$ and let $B: V \rightarrow V$ be a linear transformation. Let $\beta=\left\{e_{i}\right\}_{i=1}^{n}$ be an orthonormal basis of $V$ and set $\varepsilon_{i}:=\left\langle e_{i}, e_{i}\right\rangle$. Then the number $\sum_{i=1}^{n} \varepsilon_{i}\left\langle B\left(e_{i}\right), B\left(e_{i}\right)\right\rangle$ is independent of $\beta$.

Proof. Consider another orthonormal basis $\left\{v_{j}\right\}_{j=1}^{n}$ with $\tilde{\varepsilon}_{j}:=\left\langle v_{j}, v_{j}\right\rangle$. Let us define real numbers $b_{i j}$ by the relations

$$
e_{i}=\sum_{j=1}^{n} b_{i j} v_{j}, \quad i=1, \ldots, n .
$$


Therefore, $v_{k}=\tilde{\varepsilon}_{k} \sum_{i=1}^{n} \varepsilon_{i} b_{i k} e_{i}$. The identities $\tilde{\varepsilon}_{k}=\left\langle v_{k}, v_{k}\right\rangle= \pm 1$ and $\left\langle v_{k}, v_{j}\right\rangle=0, k \neq j$ can be written as

$$
\tilde{\varepsilon}_{k}=\sum_{i=1}^{n} \varepsilon_{i} b_{i k}^{2} \quad \text { and } \quad \sum_{i=1}^{n} \varepsilon_{i} b_{i k} b_{i j}=0, \quad j \neq k .
$$

Therefore,

$$
\begin{aligned}
\sum_{i=1}^{n} \varepsilon_{i}\left\langle B\left(e_{i}\right), B\left(e_{i}\right)\right\rangle & =\sum_{i=1}^{n} \varepsilon_{i}\left(\sum_{j, k=1}^{n} b_{i j} b_{i k}\left\langle B\left(v_{j}\right), B\left(v_{k}\right)\right\rangle\right) \\
& =\sum_{j, k=1}^{n}\left(\sum_{i=1}^{n} \varepsilon_{i} b_{i j} b_{i k}\left\langle B\left(v_{j}\right), B\left(v_{k}\right)\right\rangle\right) \\
& =\sum_{j=1}^{n}\left(\sum_{i=1}^{n} \varepsilon_{i} b_{i j}^{2}\left\langle B\left(v_{j}\right), B\left(v_{j}\right)\right\rangle\right) \\
& =\sum_{j=1}^{n} \tilde{\varepsilon}_{j}\left\langle B\left(v_{j}\right), B\left(v_{j}\right)\right\rangle
\end{aligned}
$$

as desired.

\section{THE GEODESIC SPHERES OF $\mathbf{C H}^{2}$}

In this section we first give a description of the geodesic spheres in $\mathbf{C H}^{2}$, determining its lifting to $\mathbf{Q}^{5}$. This is necessary for proving Theorem 3.3. It is also useful for carrying explicit computations.

Let $\rho>0$. Let $\widetilde{M}_{\rho}$ be the hypersurface of $\mathbf{Q}^{5}$ of all points $\left(z_{0}, z_{1}, z_{2}\right)$ such that $\left|z_{0}\right|^{2}=\cosh ^{2} \rho$ and $\left|z_{1}\right|^{2}+\left|z_{2}\right|^{2}=\sinh ^{2} \rho . \widetilde{M}_{\rho}$ is isometric to the product $S_{c}^{1} \times S_{s}^{3}$ endowed with the indefinite metric $-d s_{1}^{2}+d s_{2}^{2}$ where $S_{c}^{1}$ is the 1-dimensional Euclidean sphere with radius $c=\cosh \rho$ and $S_{s}^{3}$ is the 3-dimensional Euclidean sphere with radius $s=\sinh \rho$ and $d s_{1}^{2}, d s_{2}^{2}$ the standard metrics on $S_{c}^{1}$ and $S_{s}^{3}$.

Clearly, $\widetilde{M}_{\rho}$ is $S^{1}$-invariant, so that $\pi\left(\widetilde{M}_{\rho}\right)$ is a hypersurface of $\mathbf{C H}^{2}$, and we have

Proposition 3.1. $\pi\left(\widetilde{M}_{\rho}\right)$ is a geodesic sphere around the point $\pi((1,0,0))$ with radius $\rho$. Moreover, any geodesic sphere of $\mathbf{C H}^{2}$ is of this type, moduli an isometry of $\mathbf{C H}^{2}$ which carries the center of the geodesic sphere to $\pi((1,0,0))$. Proof. The subgroup $U(1) \times U(2)$ acts transitively on $\widetilde{M}_{\rho}$, and therefore in $\pi\left(\widetilde{M}_{\rho}\right)$. Then, given two any points $\pi(p)$ and $\pi(q)$ in $\pi\left(\widetilde{M}_{\rho}\right)$, there exists $\phi \in U(1) \times U(2)$ such that $\phi(\pi(p))=\pi(q)$. Since $\phi\left(p_{0}\right)=e^{i \theta} p_{0}$, for some $\theta$, where $p_{0}=(1,0,0)$, we have $\phi\left(\pi\left(p_{0}\right)\right)=\pi\left(p_{0}\right)$ and hence

$$
d\left(\pi(p), \pi\left(p_{0}\right)\right)=d\left(\phi(\pi(p)), \phi\left(\pi\left(p_{0}\right)\right)\right)=d\left(\pi(q), \pi\left(p_{0}\right)\right)
$$

where $d$ is the Riemannian distance in $\mathbf{C H}^{2}$. Therefore, $\pi\left(\widetilde{M}_{\rho}\right)$ is contained in the geodesic sphere centered in $\pi\left(p_{0}\right)$ and with radius $d\left(\pi(p), \pi\left(p_{0}\right)\right)$. But both $\pi\left(\widetilde{M}_{\rho}\right)$ and the geodesic sphere are compact, connected 3-manifolds so they have to coincide. 
Finally, observe that the curve $\gamma(t)=(\cosh t, \sinh t, 0)$ is a geodesic parametrized by arc length in $\mathbf{Q}^{5}$ and orthonormal to the $S^{1}$-orbits. Therefore $\pi(\gamma)$ is a geodesic in $\mathbf{C H}^{2}$, also parametrized by arc length. Since $\pi(\gamma(0))=\pi\left(p_{0}\right)$ and $\pi(\gamma(\rho)) \in \pi\left(\widetilde{M}_{\rho}\right)$, it follows that $\rho=d\left(\pi(p), \pi\left(p_{0}\right)\right)$ is the radius of $\pi\left(\widetilde{M}_{\rho}\right)$, which concludes the proof of the Proposition 3.1 since the last part of the proposition is obvious.

In this next paragraph we compute the mean curvature of the geodesic spheres as a function of its radius. These computations will be also used in $\S 5$.

From the above characterization one can also see that the geodesic spheres in $\mathbf{C H}^{2}$ are homogeneous hypersurfaces and, therefore, with constant mean curvature. To compute its mean curvature, it suffices to compute it in a point, say $\pi(p), p=(\cosh \rho, \sinh \rho, 0)$ of $\pi\left(\widetilde{M}_{\rho}\right)$. From Lemma 2.4 , we have just to compute the mean curvature $\widetilde{H}$ of $\widetilde{M}$ at $p$.

For $z$ and $w$ in $\mathbf{C}^{3}$, we consider real coordinates $z=\left(x_{1}, \ldots, x_{6}\right), w=$ $\left(y_{1}, \ldots, y_{6}\right)$ with $z_{0}=x_{1}+i x_{2}, z_{1}=x_{3}+i x_{4}$ and so on. The indefinite scalar product defined in 2.1 has the following expression in these coordinates

$$
\langle z, w\rangle=-x_{1} y_{1}-x_{2} y_{2}+\sum_{i=1}^{6} x_{i} y_{i} .
$$

Let us take the standard orthonormal basis $\left\{v_{i}\right\}_{i=1}^{6}$ of $\mathbf{R}^{6}$ with $\left\langle v_{1}, v_{1}\right\rangle=$ $\left\langle v_{2}, v_{2}\right\rangle=-1$ and $\left\langle v_{i}, v_{i}\right\rangle=1, i=3, \ldots, 6$. Then $p=c v_{1}+s v_{3}, c=$ $\cosh \rho, s=\sinh \rho$ and it is easy to see that $\left\{v_{2}, v_{4}, v_{5}, v_{6}\right\}$ is an orthonormal basis of $T_{p}\left(\widetilde{M}_{\rho}\right)$ and that $\widetilde{N}=-s v_{1}-c v_{3}$ is a unitary normal vector field of $\widetilde{M}_{\rho}$.

As before, denote by $\tilde{\nabla}$ the Riemannian connection of $\mathbf{Q}^{5}$. Then we have

$$
\left\langle\widetilde{\nabla}_{v_{i}} v_{i}, \widetilde{N}\right\rangle=\left\langle\alpha\left(v_{i}, v_{i}\right), \tilde{N}\right\rangle, \quad i=2,4,5,6,
$$

where $\alpha$ is the second fundamental form of the immersion $\widetilde{M}_{\rho} \rightarrow \mathbf{R}^{6}$. Therefore (compare with the formula for $\widetilde{H}$ in 2.4):

$$
\widetilde{H}=\frac{1}{4}\left(-\left\langle\alpha\left(v_{2}, v_{2}\right), \tilde{N}\right\rangle+\sum_{i=4}^{6}\left\langle\alpha\left(v_{i}, v_{i}\right), \tilde{N}\right\rangle\right) .
$$

Now, since $\widetilde{M}_{\rho} \rightarrow \mathbf{R}^{6}$ is a product immersion, we have $\alpha\left(v_{2}, v_{2}\right)=-\frac{1}{c} v_{1}$ and $\alpha\left(v_{i}, v_{i}\right)=-\frac{1}{s} v_{3}, i=4,5,6$. Then

$$
\widetilde{H}=\frac{1}{4}\left(-\left\langle-\frac{1}{c} v_{1},-s v_{1}-c v_{3}\right\rangle+3\left\langle-\frac{1}{s} v_{3},-s v_{1}-c v_{3}\right\rangle\right),
$$

that is

$$
\widetilde{H}=\frac{1}{4}\left(\frac{\sinh \rho}{\cosh \rho}+3 \frac{\cosh \rho}{\sinh \rho}\right)
$$

and the mean curvature $H$ of a geodesic sphere of radius $\rho$ is

$$
H=\frac{\cosh \rho}{\sinh \rho}+\frac{1}{3} \frac{\sinh \rho}{\cosh \rho} \text {. }
$$

The next result is necessary for Theorem 3.3. 
Lemma 3.2. The group generated by the reflections on totally geodesic hypersurfaces of $\mathbf{Q}^{5}$ containing the geodesic circle $\left|z_{0}\right|=1, z_{1}=z_{2}=0$ together with the $S^{1}$ subgroup act transitively on the hypersurfaces $\widetilde{M}_{\rho}$. Furthermore, the normal exponential map $\exp ^{\perp}: T(T)^{\perp} \rightarrow \mathbf{Q}^{5}$ is a diffeomorphism for any totally geodesic hypersurface $T$ of $\mathbf{Q}^{5}$ containing the circle $\left|z_{0}\right|=1$.

Proof. Let $T$ be a totally geodesic hypersurface of $\mathbf{Q}^{5}$ containing the circle $\left|z_{0}\right|=1$. We first prove that the exponential map of $\mathbf{Q}^{5}$ gives a diffeomorphism between the normal bundle of $T$ and $\mathbf{Q}^{5}$. This is equivalent to prove that any geodesic of $\mathbf{Q}^{5}$ orthogonal to $T$ has infinite length.

We observe, as it is not difficult to prove, that the totally geodesic hypersurfaces of $\mathbf{Q}^{5}$ are the intersections of hyperplanes of $\mathbf{R}^{6}$ parallel at least to one of the axis $x_{1}$ or $x_{2}$ with $\mathbf{Q}^{5}$ itself. From this, we can prove that any totally geodesic hypersurface of $\mathbf{Q}^{5}$ is a homogeneous submanifold of $\mathbf{Q}^{5}$ and that, if $T_{1}$ and $T_{2}$ are two totally geodesic hypersurfaces containing both axis $x_{1}$ and $x_{2}$ then they are congruent.

Therefore, we obtain a proof for the first part of the lemma onse we prove it for some particular totally geodesic hypersurface containing the circle mentioned above, and for some particular geodesic orthogonal to this hypersurface. But this is very easy. Choose for instance the totally geodesic hypersurface $T=\left\{\left(x_{1}, \ldots, x_{6}\right) \mid x_{3}=0\right\}$ and as an orthogonal geodesic of $\mathbf{Q}^{5}$ to $T$ the geodesic $\gamma$ given by $\gamma(t)=(\cosh t, 0, \sinh t, \ldots, 0)$. Clearly, $\gamma$ has infinite length, as required.

To conclude the proof of the lemma, we observe that the reflections on totally geodesic hypersurfaces of $\mathbf{Q}^{5}$ are the restriction of the reflections to hyperplanes of $\mathbf{R}^{6}$. Therefore, since the group generated by the reflections of $\mathbf{R}^{6}$ on hyperplanes containing the two axis $x_{1}$ and $x_{2}$ contain as a subgroup the group $U(2)$ combining this group with the $S^{1}$ action we obtain the group $U(1) \times U(2)$ which acts transitively on $\widetilde{M}_{\rho}$, proving the lemma.

Theorem 3.3. Let $M$ be a compact, embedded hypersurface with constant mean curvature of $\mathbf{C H}^{2}$. Then $M$ is a geodesic sphere.

Proof. According to what we have already seen, $\widetilde{M}=\pi^{-1}(M)$ is a compact, embedded, $S^{1}$-invariant hypersurface of $\mathbf{Q}^{5}$ with constant mean curvature.

We claim that given any totally geodesic hypersurface $T$ of $\mathbf{Q}^{5}$ which contains the axis $x_{1}$ and $x_{2}$, there exists a totally geodesic hypersurface $T^{\prime}$ of $\mathbf{Q}^{5}$ which is parallel to $T$ (that is, there exists a geodesic of $\mathbf{Q}^{5}$ orthogonal to both $T$ and $T^{\prime}$ ) such that the reflection on $T^{\prime}$ leaves $\widetilde{M}$ invariant. Therefore, since $\widetilde{M}$ is compact, all such totally geodesic hypersurfaces whose associated reflections leave $\widetilde{M}$ invariant must have a common point. Up to congruence, we may assume that this point lies on the circle $C:\left|z_{0}\right|^{2}=1, z_{1}=z_{2}=0$. It follows that $\widetilde{M}$ is invariant by the reflection on any totally geodesic hypersurface of $\mathbf{Q}^{5}$ containing the axis $x_{1}$ and $x_{2}$. Since $\widetilde{M}$ is $S^{1}$-invariant, we conclude from the previous lemma that $\widetilde{M}$ coincides with some $\widetilde{M}_{\rho}$.

We prove our claim for a totally geodesic $T$ as defined in the lemma above, the proof for another one being similar. Consider the family of totally geodesic hypersurfaces $T_{t}$ of $\mathbf{Q}^{5}$ given by

$$
T_{t}:=\left\{\left(x_{1}, \ldots, x_{6}\right) \in \mathbf{Q}^{5} \mid x_{3}=t\right\} .
$$


Clearly, this family foliates $\mathbf{Q}^{5}$. Since $\widetilde{M}$ is compact, it follows from the previous lemma that there exists $t$ large enough such that $\widetilde{M} \cap T_{t}=\varnothing$. Therefore we can apply the same method introduced by Alexandrov to conclude that $\widetilde{M}$ is invariant by some $T_{t_{0}}$. Since this holds for any foliation which contains a totally geodesic hypersurface containing the circle $C$, it follows by compacity that all those hypersurfaces whose reflexion leaves $\widetilde{M}$ invariant have a common point which has to belong to $C$, and this proves the theorem.

\section{THE HOROSPHERES OF $\mathrm{CH}^{2}$}

Let $\gamma$ be a geodesic parametrized by arc length in $\mathbf{C H}^{2}$. Given $t \in \mathbf{R}$, denote by $S_{t}$ the geodesic sphere of $\mathbf{C H}^{2}$ with center at $\gamma(t)$ and with radius $t$.

4.1 Definition. The hypersurface $L$ of $\mathbf{C H}^{2}$ given by the limit of the geodesic spheres $S_{t}$ when $t$ goes to infinity is called a horosphere of $\mathbf{C H}^{2}$.

In the next result we prove, in particular, that the horospheres are actually differentiable hypersurfaces of $\mathbf{C H}^{2}$; a useful characterization of them in terms of their lift in $\mathbf{Q}^{5}$ is also given. We observe that, up to congruences, there exists only one horosphere. This follows by observing that since $\mathbf{C H}^{2}$ is a two point homogeneous space, given two points $p_{1}, p_{2}$ of $\mathbf{C H}^{2}$ and given two geodesics $\gamma_{1}, \gamma_{2}$ through these points, there exists an isometry taking $p_{1}$ into $p_{2}$ and $\gamma_{1}$ into $\gamma_{2}$.

Proposition 4.2. Up to a congruence, a horosphere $L$ of $\mathbf{C H}^{2}$ is the projection, via $\pi$, of the hypersurface $\widetilde{L}$ of $\mathbf{Q}^{5}$ consisting of all points $\left(z_{0}, z_{1}, z_{2}\right) \in \mathbf{Q}^{5}$ satisfying the equation $\left|z_{0}+z_{1}\right|=1$.

Proof. Let $\left\{S_{t}\right\}$ be a family of geodesic spheres converging to $L$ as in the previous definition. Up to a congruence, we may assume that $S_{t}$ is centered at $\pi(\gamma(t))$ where $\gamma(t)=(\cosh t, 0, \sinh t, 0,0,0)$. Therefore $\widetilde{S}_{t}=\pi^{-1}\left(S_{t}\right)$ where $\widetilde{S}_{t}$ is given by

$$
\begin{aligned}
\widetilde{S}_{t}:=\left\{\left(\cosh (-t) w_{0}+\sinh (-t) w_{1}, \sinh (-t) w_{0}+\cosh (-t) w_{1}, w_{2}\right) \mid\right. \\
\left.\left(w_{0}, w_{1}, w_{2}\right) \in \mathbf{Q}^{5},\left|w_{0}\right|^{2}=\cosh ^{2} t,\left|w_{1}\right|^{2}+\left|w_{2}\right|^{2}=\sinh ^{2} t\right\} .
\end{aligned}
$$

To see this, observe that the set $R_{t}$ of points $\left(w_{0}, w_{1}, w_{2}\right)$ of $\mathbf{Q}^{5}$ satisfying the equation

$$
\left|w_{0}\right|^{2}=\cosh ^{2} t, \quad\left|w_{1}\right|^{2}+\left|w_{2}\right|^{2}=\sinh ^{2} t
$$

is the lift of a geodesic sphere of $\mathbf{C H}^{2}$ with radius $t$ centered at the point $\pi(\gamma(0))$. Observe also that $\widetilde{S}_{t}=\phi_{t}\left(R_{t}\right)$, where $\phi_{t}$ is the isometry

$$
\phi_{t}:=\left(\begin{array}{ccc}
\cosh (-t) & \sinh (-t) & 0 \\
\sinh (-t) & \cosh (-t) & 0 \\
0 & 0 & 1
\end{array}\right) .
$$

Since $\phi_{t}$ carries the point $\gamma(t)$ over $\gamma(0)$, we have $\widetilde{S}_{t}=\pi^{-1}\left(S_{t}\right)$.

By setting

$$
z_{0}=\cosh (-t) w_{0}+\sinh (-t) w_{1}
$$


and

we obtain

$$
z_{1}=\sinh (-t) w_{0}+\cosh (-t) w_{1}
$$

$$
w_{0}=z_{0} \cosh (-t)-z_{1} \sinh (-t) \text {. }
$$

Replacing this expression for $w_{0}$ in the equation $\left|w_{0}\right|^{2}=\cosh ^{2} t$ we get

$$
\cosh ^{2}(t)\left|z_{0}\right|^{2}+\sinh ^{2}(t)\left|z_{1}\right|^{2}+\sinh (t) \cosh (t)\left(z_{0} \bar{z}_{1}+z_{1} \bar{z}_{0}\right)=\cosh ^{2}(t) .
$$

Dividing by $\cosh ^{2}(t)$, taking the limit as $t \rightarrow \infty$ and observing that

$$
\lim _{t \rightarrow \infty} \frac{\sinh t}{\cosh t}=1
$$

we obtain the desired equation.

Theorem 4.3. The horospheres of the complex hyperbolic space $\mathbf{C H}^{2}$ are hypersurfaces with constant mean curvature equal to $4 / 3$ and are all stable. Furthermore, they are the orbits of the Heisenberg group (a 3-dimensional noncommutative nilpotent Lie group) which acts by isometries on $\mathbf{C H}^{2}$ without fixed points (therefore, the horospheres inherit a natural Lie group structure). In particular, the horospheres are (extrinsically) homogeneous submanifolds of $\mathbf{C H}^{2}$. Any two horospheres are congruent.

Proof. We have already observed above that two horospheres are congruent. Let us prove that the mean curvature of a horosphere is $4 / 3$. The computations that follow will also be important for determining the stability of the horospheres. We remark that the mean curvature of a geodesic sphere of radius $t$ in $\mathbf{C H}^{2}$ is $\sinh t /(3 \cosh t)+\cosh t / \sinh t$ which converges to $4 / 3$ as $t$ goes to infinity.

According to Lemmas 4.2 and 2.4, we have just to prove that the mean curvature of the hypersurface $\widetilde{L}$ of $\mathbf{Q}^{5}$ given by $\left|z_{0}+z_{1}\right|=1$ is 1 . We introduce a function $f: \mathbf{Q}^{5} \rightarrow \mathbf{R}$ by setting $f\left(z_{0}, z_{1}, z_{2}\right)=\left|z_{0}+z_{1}\right|^{2}$.

Since $\widetilde{L}=f^{-1}(1)$, the mean curvature $H$ of $\widetilde{L}$ is given by (see [O2, p. 124]),

$$
4 H=-\operatorname{div}_{\mathbf{Q}^{s}}\left(\frac{(\operatorname{grad}(f))_{\mathbf{Q}^{s}}}{|\operatorname{grad}(f)|_{\mathbf{Q}^{s}}}\right) .
$$

Here, $\operatorname{div}_{\mathbf{Q}^{5}}$ and $\operatorname{grad}_{\mathbf{Q}^{5}}$ are the divergence and the gradient in $\mathbf{Q}^{5}$.

$f$ can be considered as a function defined in $\mathbf{R}^{6}$. In the real coordinates $x_{1}, \ldots, x_{6}$, it is given by

$$
f\left(x_{1}, \ldots, x_{6}\right)=\left(x_{1}+x_{3}\right)^{2}+\left(x_{2}+x_{4}\right)^{2} .
$$

Therefore

$$
(\operatorname{grad}(f))_{\mathbf{R}^{6}}=\left(-2\left(x_{1}+x_{3}\right),-2\left(x_{2}+x_{4}\right), 2\left(x_{1}+x_{3}\right), 2\left(x_{2}+x_{4}\right), 0,0\right)
$$

since $d f(v)=\langle\operatorname{grad}(f), v\rangle$ implies $(\operatorname{grad}(f))_{\mathbf{R}^{6}}=\sum_{i=1}^{6} \varepsilon_{i} d f\left(e_{i}\right) e_{i}$ if $\left\{e_{i}\right\}_{i=1}^{6}$ is an orthonormal basis of $\mathbf{R}^{6}$.

At a point $z$ in $\mathbf{Q}^{5}$ consider the orthonormal basis $\left\{e_{1}, \ldots, e_{4}, i z\right\}$ of $T_{z}\left(\mathbf{Q}^{5}\right)$. Then $\left\{e_{1}, \ldots, e_{4}, i z, z\right\}$ is an orthonormal basis of $\mathbf{R}^{6}$, and we have

$$
(\operatorname{grad}(f))_{\mathbf{R}^{6}}=\sum_{i=1}^{4} d f\left(e_{i}\right) e_{i}-d f(i z) i z-d f(z) z
$$


that is

$$
(\operatorname{grad}(f))_{\mathbf{R}^{6}}=(\operatorname{grad}(f))_{\mathbf{Q}^{s}}-d f(z) z
$$

Therefore,

$$
(\operatorname{grad}(f))_{\mathbf{Q}^{5}}=2\left(-l+f x_{1},-h+2 f, l+f x_{3}, h+f x_{4}, f x_{5}, f x_{6}\right)
$$

where, for simplicity, we denote $l\left(x_{1}, x_{3}\right)=x_{1}+x_{3}, h\left(x_{2}, x_{4}\right)=x_{2}+x_{4}$, and $f\left(x_{1}, x_{2}, x_{3}, x_{4}\right)=\left(x_{1}+x_{3}\right)^{2}+\left(x_{2}+x_{4}\right)^{2}=l^{2}\left(x_{1}, x_{3}\right)+h^{2}\left(x_{2}, x_{4}\right)$.

We have that $\left\langle(\operatorname{grad}(f))_{\mathbf{Q}^{5}},(\operatorname{grad}(f))_{\mathbf{Q}^{s}}\right\rangle=4 f^{2}$ and in the points of $M$ we have $\left|(\operatorname{grad}(f))_{\mathbf{Q}^{s}}\right|^{2}=4$.

For a vector field

$$
X:=\frac{(\operatorname{grad}(f))_{\mathbf{Q}^{s}}}{\left|(\operatorname{grad}(f))_{\mathbf{Q}^{s}}\right|}=\frac{(\operatorname{grad}(f))_{\mathbf{Q}^{s}}}{2 f}
$$

we recall that

$$
\begin{aligned}
\operatorname{div}_{\mathbf{Q}^{s}}(X) & =\sum_{i=1}^{4}\left\langle\widetilde{\nabla}_{e_{i}} X, e_{i}\right\rangle-\left\langle\widetilde{\nabla}_{i z} X, i z\right\rangle \\
& =\sum_{i=1}^{4}\left\langle\nabla_{e_{i}} X, e_{i}\right\rangle-\left\langle\nabla_{i z} X, i z\right\rangle
\end{aligned}
$$

where $\widetilde{\nabla}$ and $\nabla$ are the semi-Riemannian connection of $\mathbf{Q}^{5}$ and $\mathbf{R}^{6}$ respectively. $X$ can be extended, in a natural way, to a vector field on $\mathbf{R}^{6}$, also denoted by $X$, and

$$
\operatorname{div}_{\mathbf{R}^{6}}(X)=\operatorname{div}_{\mathbb{Q}^{5}}(X)-\left\langle\nabla_{z} X, z\right\rangle
$$

or

(a)

$$
\operatorname{div}_{\mathbf{Q}^{s}}(X)=\operatorname{div}_{\mathbf{R}^{6}}(X)+\langle d(X)(z), z\rangle .
$$

On the other hand, considering $\operatorname{grad}(f)_{\mathbf{Q}^{s}}$ as a map from $\mathbf{R}^{6}$ to $\mathbf{R}^{6}$, its derivative $d\left(\operatorname{grad}(f)_{Q^{s}}\right)$ is given by the matrix

$$
2\left(\begin{array}{cccccc}
2 l x_{1}-1+f & 2 h x_{1} & 2 l x_{1}-1 & 2 h x_{1} & 0 & 0 \\
2 l x_{2} & 2 h x_{2}-l+f & 2 l x_{2} & 2 h x_{2}-1 & 0 & 0 \\
1+2 l x_{3} & 2 h x_{3} & 1+2 l x_{3}+f & 2 h x_{3} & 0 & 0 \\
2 l x_{4} & 1+2 h x_{4} & f 2 l x_{4} & 1+2 h x_{4}+f & 0 & 0 \\
2 l x_{5} & 2 h x_{5} & 2 l x_{5} & 2 h x_{5} & f & 0 \\
2 l x_{6} & 2 h x_{6} & 2 l x_{6} & 2 h x_{6} & 0 & f
\end{array}\right)
$$

so that

(b) $\left\langle d(\operatorname{grad}(f))_{\mathbf{Q}^{s}}(z), z\right\rangle=-4 f$ and $\left.\langle d X(z), z\rangle\right|_{M}=-4 / 2=-2$.

Moreover,

(c)

$$
\begin{aligned}
\operatorname{div}_{\mathbf{R}^{6}}(X)=\frac{1}{f}( & -1+2 l x_{1}+f-1+2 h x_{2}+f+1 \\
& \left.+2 l x_{3}+f+1+2 h x_{4}+f+3 f\right) \\
& +\left(-2 l+2 f x_{1}\right)\left(-l / f^{2}\right)+\left(-2 h+2 f x_{2}\right)\left(-h / f^{2}\right) \\
& +\left(2 l+2 f x_{3}\right)\left(-l / f^{2}\right)+\left(2 h+2 f x_{4}\right)\left(-h / f^{2}\right)
\end{aligned}
$$


From (a), (b) and (c) it follows that $H=1$ as required.

We will prove now that $\operatorname{Ricc}_{\mathbf{Q}^{5}}(\widetilde{N})+\|\widetilde{B}\|^{2}=0$ for the hypersurface $\widetilde{L}$ of $\mathbf{Q}^{5}$. From $\S 2.5$, it will follow that the horospheres are stable.

Observe that in this case $\widetilde{N}$ is the restriction of the following vector field of $\mathbf{R}^{6}$, also denoted by $\widetilde{N}$,

$$
\tilde{N}\left(x_{1}, \ldots, x_{6}\right)=\left(x_{1}-l, x_{2}-h, x_{3}+l, x_{4}+h, f x_{5}, f x_{6}\right) .
$$

The matrix of $d \tilde{N}$ in the standard basis $\left\{e_{i}\right\}_{i=1}^{6}$ of $\mathbf{R}^{6}$ with $\left\langle e_{1}, e_{1}\right\rangle=$ $\left\langle e_{2}, e_{2}\right\rangle=-1$ is given by

$$
d \tilde{N}=\left(\begin{array}{cccccc}
0 & 0 & -1 & 0 & 0 & 0 \\
0 & 0 & 0 & -1 & 0 & 0 \\
1 & 0 & 2 & 0 & 0 & 0 \\
0 & 1 & 0 & 2 & 0 & 0 \\
0 & 0 & 0 & 0 & 1 & 0 \\
0 & 0 & 0 & 0 & 0 & 1
\end{array}\right)
$$

Set $\bar{B}=d \tilde{N}$. Then $\widetilde{B}=\left.\bar{B}\right|_{\tilde{L}}$. We have

$$
\|\bar{B}\|_{\mathbb{R}^{6}}^{2}=-\left\langle\bar{B}\left(e_{1}\right), \bar{B}\left(e_{1}\right)\right\rangle-\left\langle\bar{B}\left(e_{2}\right), \bar{B}\left(e_{2}\right)\right\rangle+\sum_{i=1}^{6}\left\langle\bar{B}\left(e_{i}\right), \bar{B}\left(e_{i}\right)\right\rangle=6 .
$$

Since the norm of $\bar{B}$ independs on the given orthonormal basis, we can choose an orthonormal basis of $T_{z}(\widetilde{L})$, for $z \in \widetilde{L}$ and add to this basis the vectors $z$ and $\tilde{N}$ to obtain an orthonormal basis of $\mathbf{R}^{6}$. Therefore, we obtain

$$
\|\bar{B}\|_{\mathbf{R}^{6}}^{2}=\|\tilde{B}\|^{2}-\langle d \tilde{N}(z), d \tilde{N}(z)\rangle+\langle d \tilde{N}(\tilde{N}), d \tilde{N}(\tilde{N})\rangle=\|\widetilde{B}\|^{2}-1+3
$$

so that $\|\widetilde{B}\|^{2}=6+1-3=4$.

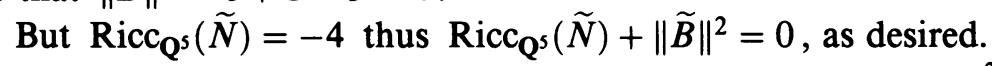

Let us describe now the (full) subgroup of isometries of $\mathbf{C H}^{2}$ which leaves invariant a horosphere of $\mathbf{C H}^{2}$. As before, we work just in $\mathbf{Q}^{5}$, by taking the lift $\widetilde{L}$ of a horosphere of $\mathbf{C H}^{2}$. Up to a congruence, we may assume that $\widetilde{L}$ is given by

$$
\left|z_{0}+z_{1}\right|=1
$$

We will determine the Killing fields $X$ of $\mathbf{Q}^{5}$ belonging to $u(1,2)$ such that $X(z) \in T_{z}(\widetilde{L})$, for any $z \in \widetilde{L}$. These Killing fields will constitute a Lie subalgebra of the Lie algebra $u(1,2)$ of the group $U(1,2)$ whose associated Lie group is the largest Lie subgroup of $U(1,2)$ acting in $\widetilde{L}$.

We recall that $u(1,2)$ is given by

$$
u(1,2)=\left\{\left(\begin{array}{ccc}
i \alpha & u & v \\
\bar{u} & i \beta & w \\
\bar{v} & \bar{w} & i \gamma
\end{array}\right), \alpha, \beta, \gamma \in \mathbf{R}, u, v, w \in \mathbf{C}\right\} .
$$

Furthermore, if $X \in u(1,2)$, then $X$ determines a Killing vector field of $\mathbf{Q}^{5}$ by setting $X(z)=X \cdot z^{t}$ where $\cdot$ denotes the usual product of matrices. Any Killing field of $\mathbf{Q}^{5}$ which is $S^{1}$-invariant is projected, via $\pi$, in a Killing field on $\mathbf{C H}^{2}$ and all Killing fields of $\mathbf{C H}^{2}$ are obtained in this way. 
Observe that a vector field $X=\left(X_{0}, X_{1}, X_{2}\right)$ of $\mathbf{Q}^{5}$ is a vector field of $\widetilde{L}$ iff it satisfies

$$
\left(X_{0}+X_{1}\right)\left(\bar{z}_{0}+\bar{z}_{1}\right)+\left(z_{0}+z_{1}\right)\left(\bar{X}_{0}+\bar{X}_{1}\right)=0
$$

for any $\left(z_{0}, z_{1}, z_{2}\right) \in \widetilde{L}$; this equation is obtained simply by derivating the equation $(*)$ along a curve on $\widetilde{L}$.

Therefore, if

$$
X=\left(\begin{array}{ccc}
i \alpha & u & v \\
\bar{u} & i \beta & w \\
\bar{v} & \bar{w} & i \gamma
\end{array}\right)
$$

then $X$ is a Killing field on $\tilde{L}$ iff

$$
\begin{gathered}
(u+\bar{u})\left(\left|z_{0}\right|^{2}+\left|z_{1}\right|^{2}\right)+z_{0} \bar{z}_{1}(2 \bar{u}+i(\alpha-\beta))+\bar{z}_{0} z_{1}(2 u+i(\beta-\alpha)) \\
+\left(z_{2} \bar{z}_{0}+z_{2} \bar{z}_{1}\right)(w+v)+\left(z_{0} \bar{z}_{2}+\bar{z}_{2} z_{1}\right)(\bar{w}+\bar{v})=0
\end{gathered}
$$

Taking the point $z_{0}=1, z_{1}=z_{2}=0$, which is a point of $\widetilde{L}$ in $(* *)$, we obtain $\bar{u}+u=0$, that is $u=i x$ for some $x \in \mathbf{R}$.

Now, any point of the type $\left(z_{0}, z_{1}, 0\right)$ of $\widetilde{L}$ satisfies the equation $\bar{z}_{0} z_{1}=$ $-\left(z_{0} \bar{z}_{1}+2\left|z_{1}\right|^{2}\right)$. Replacing $\bar{z}_{0} z_{1}$ given by this equality in $(* *)$, we obtain

$$
(2 x+\beta-\alpha)\left(\left|z_{1}\right|^{2}+z_{0} \bar{z}_{1}\right)=0 .
$$

Since we can take $\left(z_{0}, z_{1}, 0\right)$ in $\widetilde{L}$ with $\left|z_{1}\right|^{2}+z_{0} \bar{z}_{1} \neq 0$, we have $2 x+$ $\beta-\alpha=0$. Then $u=(1 / 2) i(\alpha-\beta)$. Finally, taking the points $z_{0}=-3 / 2$, $z_{1}=1 / 2, z_{2}=i$ and $z_{0}=-3 / 2, z_{1}=1 / 2, z_{2}=1$, which are points of $\widetilde{L}$, in $(* *)$, we obtain $v+w=0$.

Summarizing all these facts, we obtain that the set $\mathscr{R}$ of vectors of $u(1,2)$ whose associated Killing fields are vector fields of $\widetilde{L}$ is given by

$$
\mathscr{R}=\left\{\left(\begin{array}{ccc}
i \alpha & i \frac{\alpha-\beta}{2} & r+i s \\
-i \frac{\alpha-\beta}{2} & i \beta & -r-i s \\
r-i s & r-i s & i \gamma
\end{array}\right), \alpha, \beta, \gamma, r, s \in \mathbf{R}\right\} .
$$

By construction, $\mathscr{R}$ is a Lie subalgebra of $u(1,2)$ which, as it is immediate to see, has dimension 5. The Lie subalgebra of the isotropy subgroup of the Lie group $R$ associated to $\mathscr{R}$, corresponding to the point $(1,0,0)$ of $M$, is constituted by the vectors $X$ in $\mathscr{R}$ such that $X \cdot(1,0,0)=0$. This subalgebra is generated, as it is easy to see, by the matrix of $\mathscr{R}$ having $\alpha=\beta=r=s=0$ and $\gamma=1$. Therefore, the orbit of the point $(1,0,0)$ under $R$ has dimension 4. Since both this orbit and $\widetilde{L}$ are complete and connected, $\widetilde{L}$ with dimension 4, they must coincide.

Observe that the subspace $\tilde{\mathscr{G}}$ of $\mathscr{R}$ given by $\gamma=0$ is a Lie subalgebra of $\mathscr{R}$ and the associated Lie group, say $\widetilde{G}$, acts transitively on $\widetilde{L}$ without fixed points. This induces on $\widetilde{L}$ a natural structure of a Lie group.

As we have seen before, $L:=\widetilde{L} / S^{1}$ is a horosphere of $\mathbf{C H}^{2}$, and, by the previous construction, $R$ acts on $L$ since it contains $S^{1}$ as a subgroup. Clearly, this action is transitive. Since $S^{1}$ is in the center of $R$, and since the action of $S^{1}$ on $L$ is trivial, $R / S^{1}$ is a Lie group which still acts transitively on $L$. The Lie algebra of $R / S^{1}$ is naturally identified with the Lie subalgebra of $\mathscr{R}$ such that $\alpha+\beta+\gamma=0$. As an intersection of two Lie algebras, the 
subspace $\mathscr{G}$ of $\mathscr{R}$ given by $\gamma=0$ and $\alpha+\beta=0$ is a Lie subalgebra of $\mathscr{R}$ and, via the above identification, is a Lie subalgebra of the Lie algebra of $R / S^{1}$. The associated Lie group $G$ of $\mathscr{G}$ is a 3-dimensional Lie group acting transitively on $L$ and without fixed points, inducing therefore a structure of Lie group on the horosphere $L$. We claim that $G$ is the Heisenberg's group. In fact, straightforward computations show that $\mathscr{G}$ is noncommutative and that its derivative Lie subalgebra $[\mathscr{G}, \mathscr{G}]$ coincides with the center of $\mathscr{G}$, that is, $\mathscr{G}$ is a nilpotent 3-dimensional noncommutative Lie algebra, that is, it is the Lie algebra of the Heisenberg group.

\section{EQUIDISTANT HYPERSURFACES}

As it is well known, the complex hyperbolic space $\mathbf{C} \mathbf{H}^{2}$ is a complex manifold and the image of any complex line of the tangent space of $\mathrm{CH}^{2}$ at any point under the exponential map is a totally geodesic submanifold. We observe that two totally geodesic complex hypersurfaces $T_{1}$ and $T_{2}$ of $\mathbf{C H}^{2}$ coincide up to an isometry of $\mathbf{C H}^{2}$. In fact: since $\mathbf{C H}^{2}$ is a homogeneous manifold, we can assume that they have a point of intersection, say $p$. Moreover, it is known that the isotropy group of the isometry group of $\mathbf{C H}^{2}$ at any point is the group $U(2)$ which acts transitively on the complex lines through the origin of the tangent space at the point. Hence, up to an isometry we may assume that $T_{1}$ and $T_{2}$ have the same tangent plane at $p$. Since they are totally geodesic, they have to coincide.

By definition, an equidistant hypersurface of $\mathbf{C H}^{2}$ is the boundary of a tubular neighborhood around a totally geodesic complex hypersurface of $\mathbf{C H}^{2}$.

We want to describe now the lift to $\mathbf{Q}^{5}$ of a equidistant hypersurface of $\mathbf{C H}^{2}$. We need one lemma. We will say that a hypersurface $P$ of a Riemannian manifold $N$ is an equidistant hypersurface of a submanifold $M$ of $N$ if $P$ is contained in the boundary of a tubular neighborhood of $M$ in $N$.

Lemma 5.1. Let $N$ be a complete Riemannian manifold and let $G$ be a Lie subgroup of the isometry group of $N$ acting on $N$ with cohomogeneity one. Assume that the orbits of $G$ are properly embedded in $N$. Then, any principal orbit of $G$ is an equidistant hypersurface of any orbit of $G$.

Proof. Let $P$ be a principal orbit of $G$ and $O$ any orbit of $G$. Choose $p$ in $P$. Since $O$ is properly embedded, there exists $q \in O$ such that $s:=d(p, O)=$ $d(p, q)>0$, where $d$ denotes the Riemannian distance in $N$. Therefore, there exists $v \in T_{q}(O)^{\perp},\|v\|=s$ such that $\exp _{q} v=p$, where $\exp _{q}: T_{q}(N) \rightarrow N$ is the usual exponential map of Riemannian geometry. Hence, given $g \in G$, since $g$ is an isometry, we have $g\left(\exp _{q} v\right)=\exp _{g(q)} g_{*}(v)$ with $g_{*}(v) \in T_{g(p)}(O)^{\perp}$, $\left\|g_{*}(v)\right\|=s$. This proves that $P=G(p)$ is contained in a tubular neighborhood of radius $s$ of $O$, proving the lemma.

We can prove now the following proposition.

Proposition 5.2. Given $c \geq 0$, let $\widetilde{P}_{c}$ be the hypersurface of $\mathbf{Q}^{5}$ defined as the set of all points $\left(z_{0}, z_{1}, z_{2}\right)$ in $\mathbf{Q}^{5}$ such that $\left|z_{2}\right|^{2}=c^{2}$ (therefore, $-\left|z_{0}\right|^{2}+\left|z_{1}\right|^{2}=$ $\left.-\left(1+c^{2}\right)\right)$. Then $T:=\pi\left(\widetilde{P}_{0}\right)$ is a totally geodesic surface of $\mathbf{C H}^{2}$ isometric to the 2-dimensional hyperbolic space, and any $P_{c}:=\pi\left(\widetilde{P}_{c}\right)$ is the boundary of $a$ tubular neighborhood of radius $\sinh ^{-1}(c)$ of $T$. 
Proof. Straightforward computations show that $T$ is a totally geodesic surface in $\mathbf{C H}^{2}$ isometric to the 2-dimensional hyperbolic space.

We observe that the hypersurfaces $P_{c}, c>0$, are principal orbits and $T$ a singular orbit of the action on $\mathbf{C H}^{2}$ (via $\pi$ ) of the subgroup $U(1,1) \times U(1)$ of $U(1,2)$ consisting of the matrices of the form

$$
\left(\begin{array}{cc}
B & 0 \\
0 & e^{i \theta}
\end{array}\right)
$$

where $B \in U(1,1)$ and $\theta \in[0,2 \pi]$, so that we can apply the previous lemma to conclude that the hypersurfaces $P_{c}$ are equidistant hypersurfaces around $T$.

Finally, observe that the geodesic $\pi \circ \gamma: \mathbf{R} \rightarrow \mathbf{C H}^{2}$ where $\gamma(t)=(\cosh (t), 0$, $0,0, \sinh (t), 0)$ is orthogonal to $T$ at $t=0$, is parametrized by arc length, and satisfies $\gamma(t) \in P_{\sinh ^{2}(t)}$, concluding the proof of the proposition.

Theorem 5.3. An equidistant hypersurface $P_{c}$ in $\mathbf{C H}^{2}$ is a homogeneous hypersurface with constant mean curvature $\left(1+4 c^{2}\right) / 3 c \sqrt{1+c^{2}}$. It is stable if $c \geq \sqrt{2} / 2$ and unstable otherwise. Two equidistant hypersurfaces are congruent iff they have the same mean curvature.

Proof. It follows from the above that the equidistant hypersurfaces are homogeneous hypersurfaces of $\mathbf{C H}^{2}$. Let us compute now their mean curvature. It is sufficient to compute the mean curvature of the lift $\widetilde{P}_{c}$ at a particular point $p$. We choose $p=\left(\sqrt{1+c^{2}}, 0,0,0, c, 0\right)$. We have

$$
p=\sqrt{1+c^{2}} v_{1}+c v_{5}
$$

where $\left\{v_{i}\right\}_{i=1}^{6}$ is the standard orthonormal basis of $\mathbf{R}^{6}$ with $\left\langle v_{1}, v_{1}\right\rangle=\left\langle v_{2}, v_{2}\right\rangle$ $=-1$ and $\left\langle v_{i}, v_{i}\right\rangle=1, i=3,4,5,6$. The set $\left\{v_{2}, v_{3}, v_{4}, v_{6}\right\}$ is an orthonormal basis of $T_{p}\left(\widetilde{P}_{c}\right)$, and it is not difficult to prove that $N:=-c v_{1}-$ $\sqrt{1+c^{2}} v_{5}$ is unitary and normal to $\widetilde{P}_{c}$ in $\mathbf{Q}^{5}$.

As in the case of the geodesic spheres (see $\S 3$ ), we have

$$
\widetilde{H}_{p}=\frac{1}{4}\left(\sum_{i=2}^{4}\left(\varepsilon_{i}\left(\tilde{\alpha}\left(v_{i}, v_{i}\right), N\right\rangle\right)+\left\langle\tilde{\alpha}\left(v_{6}, v_{6}\right), N\right\rangle\right)
$$

where $\tilde{\alpha}$ is the second fundamental form of $\widetilde{P}_{c}$ in $\mathbf{R}^{6}$. From the definition of $\widetilde{P}_{c}$, it follows that

$$
\tilde{\alpha}\left(v_{i}, v_{i}\right)=\frac{1}{\sqrt{1+c^{2}}}\left\langle v_{i}, v_{i}\right\rangle v_{1}, \quad i=2,3,4,
$$

and

so that

$$
\tilde{\alpha}\left(v_{6}, v_{6}\right)=-\frac{1}{c} v_{5}
$$

$$
\widetilde{H}_{p}=\frac{1}{4}\left(\frac{1+4 c^{2}}{c \sqrt{1+c^{2}}}\right)
$$

hence, the mean curvature of $P_{c}$ is

$$
H=\frac{1}{3}\left(\frac{1+4 c^{2}}{c \sqrt{1+c^{2}}}\right)
$$

as required. 
We study now the stability of the $P_{c}$. To do that, we first compute the value of Ricc $\widetilde{N}+\|\widetilde{B}\|^{2}$ of the hypersurface $\widetilde{P}_{c}$ of $\mathbf{Q}^{5}$.

We have that the Ricci curvature of $\mathbf{Q}^{5}$ (with respect to any direction) is constant with value -4 (see $[02, \mathrm{p} .88])$. Since $\widetilde{P}_{c}$ is a homogeneous submanifold of $\mathbf{Q}^{5},\|\widetilde{B}\|$ is constant so that it is enough to compute its value at the point $p$ fixed above.

Using the computations above and the ones of $\S 2.5$, we obtain

$$
\|\widetilde{B}\|^{2}=\frac{3 c^{2}}{1+c^{2}}+\frac{1+c^{2}}{c^{2}}
$$

and

$$
\operatorname{Ricc}_{Q^{s}}(\tilde{N})+\|\widetilde{B}\|^{2}=\frac{1-2 c^{2}}{c^{2}\left(1+c^{2}\right)} .
$$

Thus, $c^{2} \geq \frac{1}{2}$ implies that $\operatorname{Ricc}_{\mathbf{Q}^{5}}(\tilde{N})+\|\widetilde{B}\|^{2} \leq 0$ and from 2.5 it follows that $P_{c}$ is stable in this case.

Let us assume $c^{2}<\frac{1}{2}$. To prove that $P_{c}$ is unstable it suffices to exhibit a piecewise smooth function $f$ defined in a relatively compact domain $D$ of $\widetilde{P}_{c}$ satisfying the following conditions:

$$
\begin{gathered}
f \text { is } S^{1} \text {-invariant, that is, } f\left(e^{i \theta} z\right)=f(z), \\
\left.f\right|_{\partial D} \equiv 0, \quad \int_{D} f d \widetilde{P}_{c}=0,
\end{gathered}
$$

and

$$
\mathscr{T}^{\prime \prime}(0)(f)=\int_{d}\left[|\operatorname{grad} f|^{2}-\left(\operatorname{Ricc}_{\mathbf{Q}^{s}}(N)+\|B\|^{2}\right) f^{2}\right] d \widetilde{P}_{c}<0 .
$$

To compute this integral, we use the following local coordinates of $\widetilde{P}_{c}$ :

$$
\tau:(\alpha, \beta, \gamma, t) \rightarrow\left(e^{i \alpha} A \cosh (t / A), e^{i \beta} A \sinh (t / A), e^{i \gamma} c\right)
$$

where $A=\sqrt{1+c^{2}}, \alpha, \beta$ and $\gamma$ vary in the interval $(0,2 \pi)$, and $t \in \mathbf{R}$. In these coordinates, the volume element of $\widetilde{P}_{c}$ is

$$
d \widetilde{P}_{c}=c\left(1+c^{2}\right) \cosh (t / A)|\sinh (t / A)| d t \wedge d \alpha \wedge d \beta \wedge d \gamma .
$$

For a fixed real number $a$, set $D_{a}:=\{\tau(\alpha, \beta, \gamma, t) \mid t \in(-a, a)\}$. Clearly, $D_{a}$ is a relatively compact domain in $\widetilde{P}_{c}$.

Now, for a fixed $b \in(0, a)$, consider the piecewise smooth real function $f$ defined in $[-a, a]$ by

$$
f(t)= \begin{cases}\frac{-t-a}{a-b} b & \text { if }-a \leq t \leq-b \\ t & \text { if }-b \leq t \leq b \\ \frac{-t+a}{a-b} b & \text { if } b \leq t \leq a\end{cases}
$$

$f$ determines a piecewise smooth function $\tilde{f}$ on $\bar{D}_{a}$ by setting

$$
\tilde{f}(\tau(\alpha, \beta, \gamma, t)):=f(t) .
$$


It follows that $\left.\tilde{f}\right|_{\partial D_{a}}=0$ since $f(a)=f(-a)$ and that

$$
\begin{aligned}
\int_{D_{a}} \tilde{f} d \widetilde{P}_{c} & =\left[c^{2}\left(1+c^{2}\right) \int_{\alpha, \beta, \gamma=0}^{2 \pi} d \alpha d \beta d \gamma\right] \int_{-a}^{a} f(t) \cosh (t / A)|\sinh (t / A)| d t \\
& =0
\end{aligned}
$$

the last integral being zero since $f$ is an odd function.

Now, we will calculate $\mathscr{T}^{\prime \prime}(0)(\tilde{f})$. In these coordinates, it is easy to prove that $|\operatorname{grad} \tilde{f}|^{2}=f^{\prime}(t)^{2}$.

For simplicity, set

$$
G:=c^{2}\left(1+c^{2}\right) \int_{\alpha, \beta, \gamma=0}^{2 \pi} d \alpha d \beta d \gamma=8 c^{2}\left(1+c^{2}\right) \pi^{3}
$$

and

$$
I:=\operatorname{Ricc}_{Q^{5}}(\widetilde{N})+\|\widetilde{B}\|^{2}=\frac{1-2 c^{2}}{c^{2}\left(1+c^{2}\right)} .
$$

We observe that $I>0$ since by hypothesis $c^{2}<\frac{1}{2}$. Then

$$
\begin{aligned}
\mathscr{T}^{\prime \prime}(0)(\tilde{f})= & \int_{D_{a}}\left(|\operatorname{grad}(\tilde{f})|^{2}-I \tilde{f}^{2}\right) d \widetilde{P}_{c} \\
= & G\left(\int_{-a}^{a} f^{\prime}(t)^{2} \cosh (t / A)|\sin h(t / A)| d t\right. \\
& \left.\quad-I \int_{-a}^{a} f(t)^{2} \cosh (t / A)|\sin h(t / A)| d t\right) .
\end{aligned}
$$

Since $f^{\prime}$ and $f^{2}$ are even functions, we have

$$
\begin{gathered}
\int_{-a}^{a} f^{\prime}(t)^{2} \cosh (t / A)|\sinh (t / A)| d t=2 \int_{0}^{b} \cosh (t / A) \sinh (t / A) d t \\
+\frac{2 b^{2}}{(a-b)^{2}} \int_{a}^{b} \cosh (t / A) \sinh (t / A) d t
\end{gathered}
$$

and

$$
\begin{gathered}
I \int_{-a}^{a} f(t)^{2} \cosh (t / A)|\sinh (t / A)| d t=2 I \int_{0}^{b} t^{2} \cosh \left(\frac{t}{A}\right) \sinh \left(\frac{t}{A}\right) d t \\
\quad+\frac{2 b^{2}}{(a-b)^{2}} \int_{a}^{b}(a-t)^{2} \cosh (t / A) \sinh (t / A) d t
\end{gathered}
$$

We will prove that it is possible to choose $a$ and $b$ such that (2)-(1)>0, that is, $\mathscr{T}^{\prime \prime}(0)(\tilde{f})<0$. 
We have

$$
\begin{aligned}
(2)-(1)= & 2 \int_{0}^{b}\left(I t^{2}-1\right) \cosh (t / A) \sinh (t / A) d t \\
& +\frac{2 b^{2}}{(a-b)^{2}} \int_{a}^{b}\left(I(a-t)^{2}-1\right) \cosh (t / A) \sinh (t / A) d t \\
= & 2 \int_{0}^{b}\left(I t^{2}-1\right) \cosh (t / A) \sinh (t / A) d t \\
& +\frac{2 b^{2}}{(a-b)^{2}} \int_{b}^{a-1 / \sqrt{I}}\left(I(a-t)^{2}-1\right) \cosh (t / A) \sinh (t / A) d t \\
& +\frac{2 b^{2}}{(a-b)^{2}} \int_{a-1 / \sqrt{I}}^{a}\left(I(a-t)^{2}-1\right) \cosh (t / A) \sinh (t / A) d t
\end{aligned}
$$

We observe that $I t^{2}-1>0$ if $t>1 / \sqrt{I}$ so that it is possible to choose $b$ with $b<a$ such that

$$
\int_{0}^{b}\left(I t^{2}-1\right) \cosh (t / A) \sinh (t / A) d t>0 .
$$

On the other hand, $a-1 / \sqrt{I}$ is a root of the polynomial $p(t):=I(a-t)^{2}-1$, and $p(t) \geq 0$ for $0 \leq t \leq 1 / \sqrt{I}$. Then, the second term of the last equality above is positive for all $b$ such that $0<b<a-1 / \sqrt{I}$. Since $-1 \leq p(t) \leq 0$ for $a-1 / \sqrt{I} \leq t \leq a$ we have

$$
\begin{aligned}
\int_{a-1 / \sqrt{I}}^{b} & \left(I(a-t)^{2}-1\right) \cosh (t / A) \sinh (t / A) d t \\
\geq & \int_{a-1 / \sqrt{I}}^{a}-\cosh (t / A) \sinh (t / A) d t \\
& =-\frac{A}{2}\left(\sinh ^{2}(a / A)-\sinh ^{2}((1 / A))(a-(1 / \sqrt{I}))\right) .
\end{aligned}
$$

This last term is negative but tends to zero when $a$ increases. This means that fixing $b$ with $b<a$ such that

$$
\int_{0}^{b}\left(I t^{2}-1\right) \cosh (t / A) \sinh (t / A) d t>0
$$

it is possible to choose $a$ satisfying $b<a-1 / \sqrt{I}$ with

$$
\left|\int_{a-1 / \sqrt{I}}^{a}-\cosh (t / A) \sinh (t / A) d t\right|
$$

small enough in such a way that (2)-(1) is positive.

\section{REFERENCES}

[A] A. D. Alexandrov, A characteristic property of spheres, Ann. Mat. Pura Appl. 58 (1962), 305-315.

[BdoCE] J. L. Barbosa, M. P. do Carmo, and J. Eschenburg, Stability of hypersurfaces with constant mean curvature in Riemannian manifolds, Math. Z. 197 (1988), 124-138. 
[KN] S. Kobayashi and K. Nomizu, Foundations of differential geometry, vol. II, Interscience, 1969.

[O1] B. O'Neill, The fundamental equations of a Riemannian submersion, Michigan Math. J. 23 (1966), 459-469.

[O2] _ Semi Riemannian geometry, Academic Press, 1983.

Departamento de Matemática-ICEX, Universidade Federal de Minas Gerais, CaiXa Postal 702, 30161-Belo Horizonte-MG, BraZil

Departamento de Matemática, Universidade federal do Rio de Janeiro, Rio de JANEIRO, BRAZIL

Instituto de Matemática, Universidade Federal do Rio Grande do Sul, Av. Bento Gonçalves 9500, 91500-Porto Alegre-RS, Brazil 